\title{
Influencia del acabado superficial sobre el comportamiento tribológico de capas nitrocarburadas en acero X40CrMoV5 1
}

\author{
V. Miguel*, A. Calatayud*, J. Coello*, A. Martínez* y A. Caminero* \\ Resumen En este trabajo se estudia la influencia que el acabado superficial del acero X40CrMoV5 1 \\ tiene sobre la capa blanca o de combinación formada en el proceso de la nitrocarburación \\ gaseosa. Se consideran acabados superficiales en el rango de 0,02 a 1,50 $\mu \mathrm{m}$ para Ra. Los \\ resultados obtenidos indican que la rugosidad aumenta en todos los casos, aunque tiene \\ poca influencia sobre el espesor de capa formada. También se ha investigado el \\ comportamiento frente al desgaste abrasivo de las capas obtenidas que están constituidas en \\ su totalidad por nitrocarburo $\varepsilon$ y son de un espesor aproximado de $6 \mu \mathrm{m}$. Se ha encontrado \\ un comportamiento diferenciado frente al desgaste de la zona externa de la capa frente a la \\ zona subsuperficial. Los resultados revelan que la capa externa de la capa de combinación \\ es poco efectiva frente al desgaste en relación con la capa subsuperficial. La zona de peor \\ comportamiento es mayor para las probetas con acabados superficiales más finos.
}

Palabras clave Nitrocarburación. Rozamiento. Desgaste. Acero X40CrMoV5 1. Acabado superficial.

\section{Surface texture effect on tribologic behaviour in nitrocarburized X40CrMoV5 1 steel layers}

\begin{abstract}
In this work the surface roughness behaviour in the forming of gaseous nitrocarburized layers has been analysed. Ra values from 0.02 to $1.50 \mu \mathrm{m}$ has been considered. Obtained results prove a minor influence on layer thickness. A little increase of $\mathrm{Ra}$ has been observed. The abrasive wear resistance of nitrocarburized specimens was researched. The carbonitride $\varepsilon$ is the only constituyent in the about $6 \mathrm{~mm}$ thickness layers that have been carried out in this work. The tests revealed a minor wear resistance of the external part in the layers. This way, the external zone is less effective to wear phenomena than the internal. The specimens with small roughness parameters values show a higher poor wear resistance zone.
\end{abstract}

Keywords Nitrocarburizing. Friction. Wear. X40CrMoV5 1 steel. Surface roughness.

\section{INTRODUCCIÓN}

Las actuales exigencias tecnológicas ponen de manifiesto la necesidad de disponer de aceros con elevadas prestaciones superficiales (elevada dureza, resistencia al desgaste y a la fatiga). A su vez puede ser necesaria una alta tenacidad en el resto del material. Entre los tratamientos que permiten modificar la zona superficial de los aceros, aumentando considerablemente su dureza y su resistencia al desgaste figura la nitrocarburación gaseosa. La obtención de capas nitrocarburadas con estructuras adecuadas a la aplicación en cuestión depende de la correcta selección de los parámetros que intervienen en dicho proceso ${ }^{[1]}$.

Una de las aplicaciones que demanda el empleo de aceros nitrocarburados es la fabricación de matrices para extrusión de aluminio. La capa de combinación o capa blanca debe estar constituida en su totalidad por nitrocarburo $\varepsilon$ (Fe 2,3 CN) de gran dureza $(1.500 \mathrm{HV})$ y mayor ductilidad que los nitruros presentes en otros procesos ${ }^{[2 \text { y } 3]}$.

Una variable importante que afecta al comportamiento de las capas nitrocarburadas es la temperatura de trabajo de la herramienta en la medida en que se produce la oxidación de parte de la capa

${ }^{*}$ C Ciencia e Ingeniería de Materiales. Instituto de Desarrollo Regional. Universidad de Castilla-La Mancha, Campus Universitario, 02071 Albacete, España. 
blanca y se modifica la capa de difusión situada entre aquélla y el acero base.

A pesar de que la capa blanca esté compuesta por nitrocarburo $\varepsilon$ en su totalidad, existen variaciones en su morfología que conducen a comportamientos frente al desgaste diferenciados. En procesos de nitrocarburación en baños de sales se ha justificado la existencia de capas que presentan una estructura más porosa en el exterior. Así la estructura superficial y la subsuperficial difieren en el comportamiento en fenómenos de deslizamiento ya que la porosidad favorece el atrapamiento de lubricante y disminuye el coeficiente de fricción. No obstante, se ha comprobado que la resistencia al desgaste es menor para la capa externa, más porosa, que para la subsuperficial ${ }^{[4 \text { y } 5]}$.

En cualquier caso, para unas condiciones de lubricación dadas, el acabado de la superficie tiene una influencia determinante en los mecanismos de fricción y de desgaste. A su vez, el desgaste modifica la rugosidad de la superficie por lo que se produce una evolución en el comportamiento del elemento con su uso.

El desgaste abrasivo es característico en diversos procesos de conformado de metales ${ }^{[6]}$.

En procesos de extrusión de aluminio este tipo de desgaste puede ser atribuido a la presencia de fases intermetálicas duras del tipo Al-Fe-Si, Al-Cu y $\mathrm{Al}-\mathrm{Fe}-\mathrm{Cu}-\mathrm{Si}$ entre otras. La existencia de dichas fases provocan el deterioro de la matriz.

Existe un gran número de trabajos que estudian el comportamiento de este tipo de superficies frente a mecanismos de desgaste adhesivo. Sin embargo no se encuentran referencias que investiguen el comportamiento abrasivo en las matrices. Ello se debe al hecho de que se atribuye este tipo de desgaste sólo al sufrido por el perfil extruido, justificándolo en base a la gran diferencia de dureza con respecto a la matriz. La experiencia de los autores en el comportamiento industrial de matrices nitrocarburadas revela la importancia del mecanismo de desgaste abrasivo sobre éstas como consecuencia de la presencia de las fases intermetálicas mencionadas en el material a extruir.

El fenómeno descrito es más acusado cuando la aleación de aluminio presenta una mayor concentración de impurezas que conducen a la acumulación de fases duras en la parte final del bruto. En ocasiones, esta zona no es desechada en su totalidad y es extruida en parte, lo que ocasiona una mayor presencia del fenómeno de desgaste abrasivo sobre la matriz.

En el presente trabajo analizamos la influencia de la rugosidad sobre el comportamiento frente al desgaste abrasivo del acero X40CrMoV5 1 nitrocarburado, utilizado frecuentemente en aplicaciones como las descritas con anterioridad. Para ello, se han ensayado probetas con distinto acabado superficial, investigando si existe influencia sobre la formación de capas y sobre su posterior comportamiento frente al desgaste.

\section{MATERIALES Y PROCEDIMIENTO EXPERIMEN- TAL}

El tratamiento de nitrocarburación se efectuó a $565^{\circ} \mathrm{C}$ durante $3 \mathrm{~h}$ y se llevó a cabo en una instalación experimental bajo condiciones fijadas con anterioridad $^{[1]}$ que garantizaran la obtención de una capa blanca monofásica de nitrocarburo $\varepsilon$. Se nitrocarburaron probetas de $30 \mathrm{~mm}$ de diámetro de acero $\mathrm{X} 40 \mathrm{CrMoV} 51$, previamente templadas en aire a $1.030^{\circ} \mathrm{C}$ y revenidas a $600^{\circ} \mathrm{C}$. Los tratamientos se extendieron a distintas series de probetas con rugosidades diferentes. La composición del acero se establece en la tabla I.

Los distintos acabados superficiales sobre el material de partida se realizaron mediante el empleo de papel de esmeril de distinta granulometría. Las rugosidades obtenidas se muestran en la tabla II.

Los parámetros de rugosidad se obtuvieron mediante un rugosímetro MAHR PERTHOMETER S2. Se determinaron los parámetros $\mathrm{Ra}$ y $\mathrm{Rz}$ definidos en la norma EN ISO 4288. El perfil de rugosidad sobre el que han sido obtenidos es de $12,5 \mathrm{~mm}$. Para su determinación se realizaron $4 \mathrm{me}$ diciones sobre respectivos diámetros de cada probeta; los resultados obtenidos se promediaron.

Las probetas para los ensayos de desgaste se obtuvieron cortando por la mitad los discos tratados. Dichos ensayos se efectuaron empujando la probeta con una presión de $0,08 \mathrm{MPa}$ sobre un disco que giró a $250 \mathrm{rpm}$, cubierto superficialmente con un paño DP-Dur de Struers y pasta de diamante de $6 \mu \mathrm{m}$ de granulometría; para ello se utilizó una pulidora convencional dotada de un brazo portamuestras. Se empleó lubricante DP BLUE de Struers de base etanol en los ensayos efectuados. El

Tabla I. Composición química del acero X40CrMoV5 1 Table I. X40CrMoV5 1 steel composition

\begin{tabular}{|c|c|c|c|c|c|c|c|c|}
\hline & C & $\mathrm{Si}$ & $M n$ & $P$ & $S$ & $\mathrm{Cr}$ & Mo & V \\
\hline Min & 0,37 & 0,90 & 0,30 & - & - & 4,80 & 1,20 & 0,90 \\
\hline Max & 0,43 & 1,20 & 0,50 & 0,030 & 0,030 & 5,50 & 1,50 & 1,10 \\
\hline
\end{tabular}


Tabla II. Acabado superficial del acero previo al tratamiento de nitrocarburación

Table II. Steel surface roughness before the nitrocarburizing treatment

\begin{tabular}{ccccl}
\hline Acabado superficial & Ra $(\mu \mathrm{m})$ & $\mathbf{R z}(\mu \mathrm{m})$ & Rmax. $(\mu \mathrm{m})$ & Direccionalidad \\
\hline 1 & $0,01-0,05$ & $0,08-0,2$ & $0,13-0,88$ & Sin dirección preferente \\
2 & $0,04-0,09$ & $0,4-0,7$ & $0,5-1,4$ & Sin dirección preferente \\
3 & $9,27-0,32$ & $2,8-3,3$ & $3,9-5,6$ & Sin dirección preferente \\
4 & $1,2-1,5$ & $9,2-9,6$ & $10,5-11,2$ & Sin dirección preferente \\
\hline
\end{tabular}

lubricante fue añadiéndose periódicamente, en cada minuto de ensayo.

Las condiciones del ensayo fueron fijadas tras diversas pruebas preliminares en las que se emplearon como elemento de desgaste papeles de esmeril con diversa granulometría. El desgaste provocado con diferentes lijas, hasta grano 1.000 , era excesivamente rápido y poco adecuado para la toma de datos. El empleo de lijas de menor tamaño de grano, se utilizaron lijas de grano 4000 , fue también desestimado porque se agotaban muy rápidamente. Finalmente se optó por el empleo de paño con pasta de diamante de $6 \mu \mathrm{m}$. Para garantizar la homogeneidad en los diferentes ensayos se partió siempre de un paño regenerado con una cantidad fija de pasta y de lubricante.

El ensayo a desgaste se prolonga hasta la total desaparición de capa blanca con un tiempo máximo de $60 \mathrm{~min}$.

Se comprobó que el tiempo de agotamiento del paño bajo las condiciones de desgaste indicadas era superior al de duración de los ensayos. Para ello se contrastaron ensayos realizados en distinto orden sobre un mismo paño y partiendo siempre de probetas nuevas. Lógicamente la capacidad de desgaste del paño disminuye conforme el tiempo de ensayo progresa, pero no es muy significativo, especialmente si se comparan resultados obtenidos en las primeras etapas del ensayo.

La medición de la capa blanca se efectuó mediante técnicas de análisis de imagen en la cara transversal a la superficie rozada. Para ello se interrumpió el ensayo de desgaste en intervalos de tiempo que se establecieron experimentalmente en función de la severidad del desgaste. Estos tiempos figuran en abcisas en el registro de ensayo correspondiente.

\section{RESULTADOS Y DISCUSIÓN}

Los valores de $\mathrm{Ra}$ y $\mathrm{Rz}$ antes y después de los tratamientos superficiales se establecen en la tabla III.
Tabla III. Variación de la rugosidad con el tratamiento de nitrocarburación

Table III. Surface roughness values before and after the nitrocarburizing treatment

\begin{tabular}{|c|c|c|c|c|c|c|}
\hline \multirow[t]{2}{*}{$\begin{array}{l}\text { Acabado } \\
\text { superficial }\end{array}$} & \multicolumn{2}{|c|}{$\begin{array}{c}\text { Antes del } \\
\text { tratamiento } \\
(\mu \mathrm{m})\end{array}$} & \multicolumn{2}{|c|}{$\begin{array}{c}\text { Después del } \\
\text { tratamiento } \\
(\mu \mathrm{m})\end{array}$} & \multicolumn{2}{|c|}{$\begin{array}{c}\text { Variación de } \\
\text { tratamiento } \\
\qquad(\mu \mathrm{m})\end{array}$} \\
\hline & $\mathrm{Ra}^{0}$ & $\mathrm{Rz}^{0}$ & $\mathrm{Ra}^{\mathrm{nc}}$ & $\mathrm{Rz}^{\mathrm{nc}}$ & $\mu \mathrm{Ra}^{\mathrm{nc}}$ & $\mu R z^{n c}$ \\
\hline 1 & 0,02 & 0,10 & 0,13 & 1,00 & 0,11 & 0,90 \\
\hline 1 & 0,03 & 0,19 & 0,11 & 0,84 & 0,08 & 0,65 \\
\hline 2 & 0,09 & 0,73 & 0,23 & 1,73 & 0,14 & 1,00 \\
\hline 3 & 0,27 & 2,85 & 0,39 & 3,49 & 0,12 & 0,64 \\
\hline 4 & 1,54 & 9,63 & 1,72 & 10,93 & 0,18 & 1,30 \\
\hline
\end{tabular}

Se observa un pequeño incremento de los parámetros de rugosidad de las probetas tras el tratamiento superficial.

El análisis microestructural no revela ningún indicio de que las irregularidades del perfil afecten de forma determinante al crecimiento de la capa para los acabados superficiales contemplados. Efectivamente no existe una tendencia generalizada al crecimiento basada en la existencia de picos o valles, tal y como se puede observar en la figura 1 para diversas probetas con el acabado superficial más basto; la capa de combinación alcanza mayor o menor espesor tanto en los picos como en los valles del perfil. En la figura 2 se establecen distintas micrografías comparativas de los diversos acabados superficiales. En todos los casos el espesor de la capa de combinación obtenidas estuvo comprendido entre las $6 \mu \mathrm{m}$ y $7 \mu \mathrm{m}$.

Para los ensayos de desgaste, se realizó un experimento por cada acabado superficial considerado. De la observación de las curvas de desgaste de la figura 3, se establece que en todos los ensayos existe una primera etapa en la que la velocidad de 
Influencia del acabado superficial sobre el comportamiento tribológico de capas nitrocarburadas en acero X40CrMoV5 1 V. Miguel, A. Calatayud, J. Coello, A. Martínez y A. Caminero
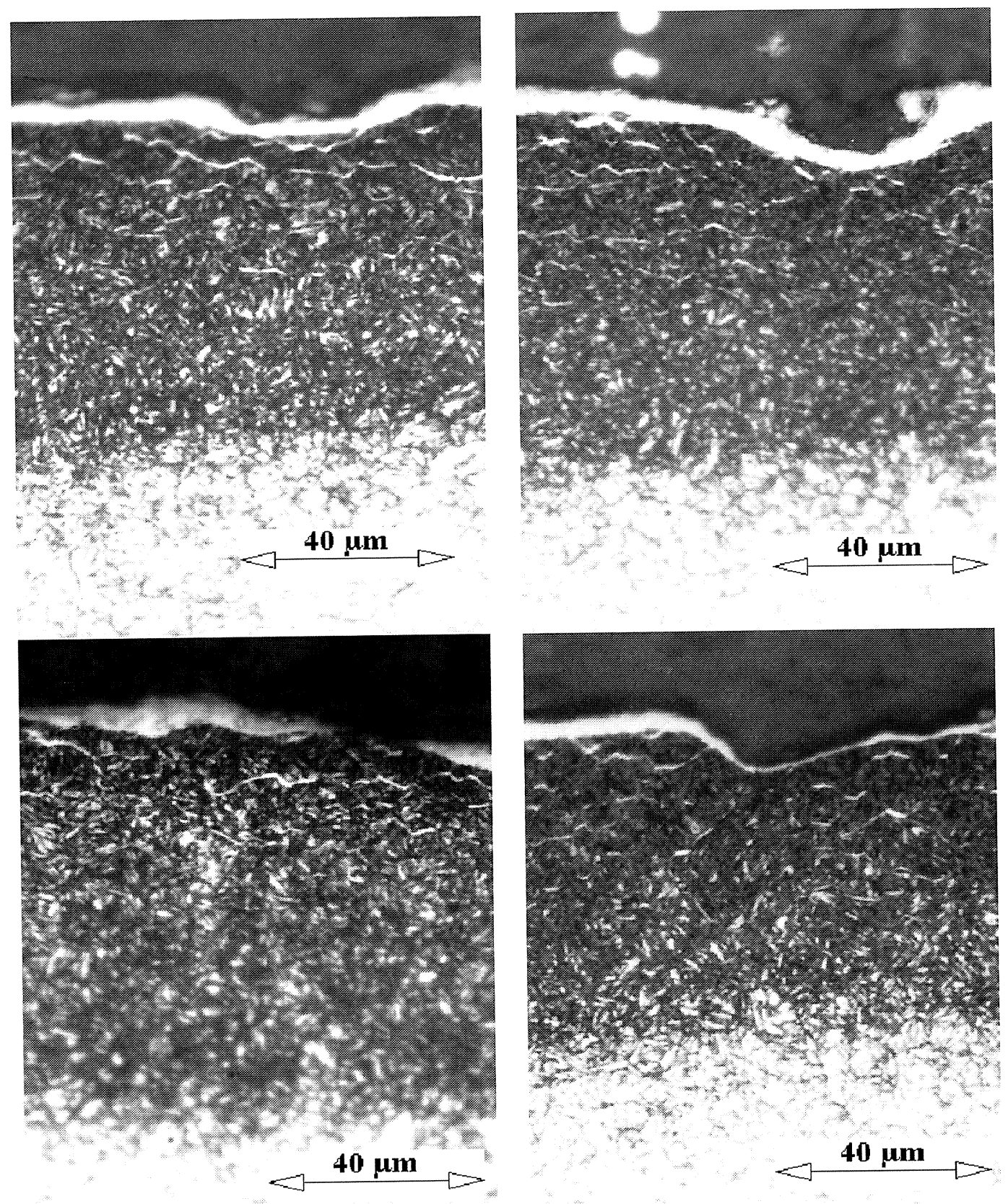

Figura 1. Micrografías de diversas probetas nitrocarburadas; ataque Nital 1. Antes del tratamiento, $\operatorname{Ra}^{0}=1,5 \mu \mathrm{m}$. Después del tratamiento, $\operatorname{Ra}^{\mathrm{nc}}=1,7 \mu \mathrm{m}$.

Figure 1. Various nitrocarburized specimens photomicrographs; etching Nital 1. Before the treatment, $R a^{0}=1.5 \mu \mathrm{m}$. After the treatment, $R a^{n c}=1.7 \mu \mathrm{m}$.

desgaste, uniforme, es mayor; posteriormente la velocidad de desgaste decrece paulatinamente con el tiempo.

El tiempo de ensayo y el espesor de capa desgastada, cuando se produce el cambio mencionado, varían en función del acabado superficial del acero. En las probetas con los acabados superficiales más finos (acabados 1 y 2 ), en las que la rugosi- dad superficial es menor, el cambio de tendencia en el desgaste ocurre cuando ha desaparecido el $90 \%$ de la capa de combinación. Para los acabados más bastos (3 y 4), esto ocurre en torno al $70 \%$. En términos de espesor los resultados corresponden a 5 $\mu \mathrm{m}$ (acabados 1 y 2 ) у $2 \mu \mathrm{m}$ (acabados 3 y 4 ), aproximadamente. Dado el comportamiento diferenciado observado en función del acabado superficial se 
Influencia del acabado superficial sobre el comportamiento tribológico de capas nitrocarburadas en acero X40CrMoV5 1 V. Miguel, A. Calatayud, J. Coello, A. Martínez y A. Caminero
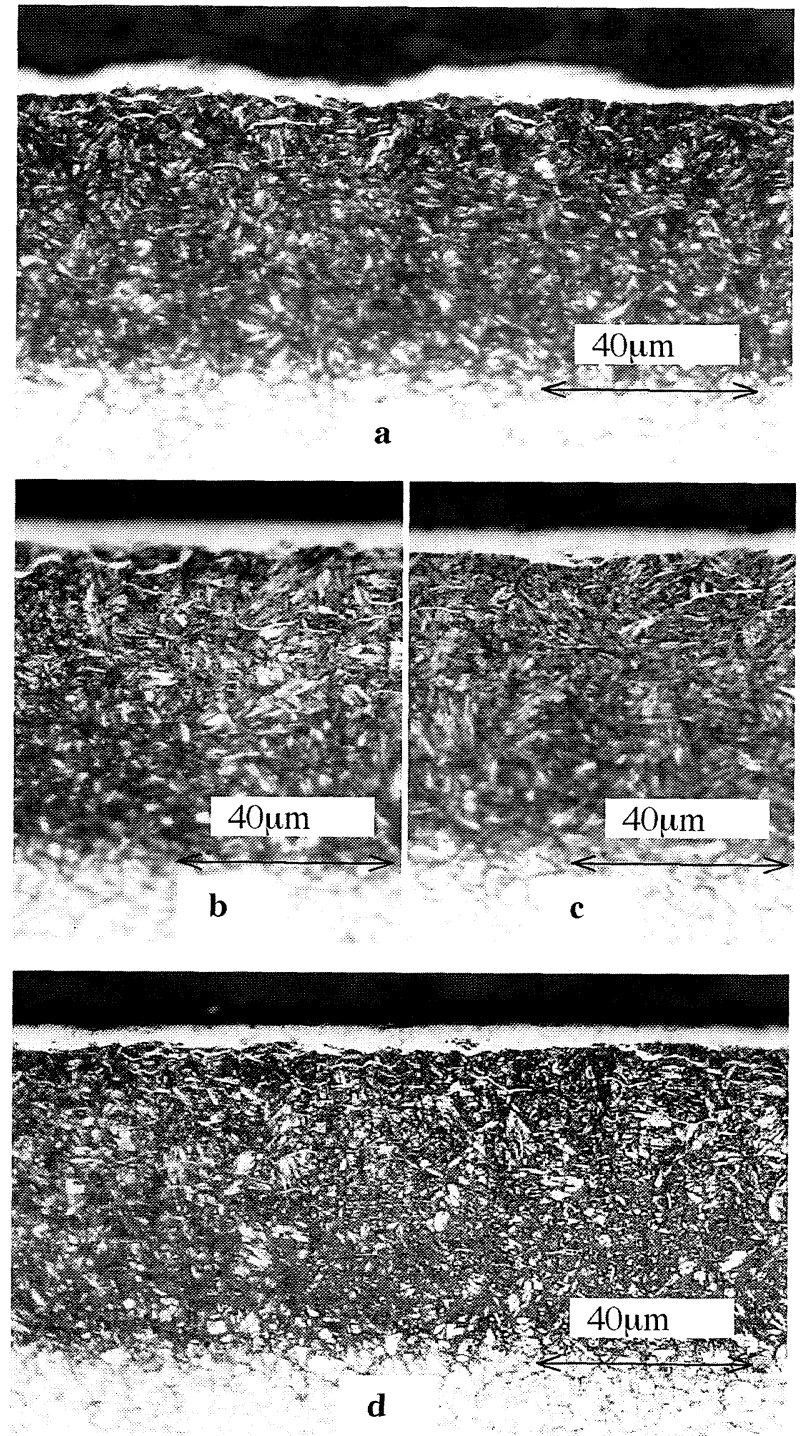

Figura 2. Micrografías de muestras nitrocarburadas con distinto acabado superficial. Antes del tratamiento de nitrocarburación, $\left(R a^{0}\right)$ y después, $\left(R a^{n c}\right)$. a) $\operatorname{Ra}^{0}=1,54 \mu \mathrm{m}$; $\mathrm{Ra}^{\mathrm{nc}}=1,72 \mu \mathrm{m}$. b) $\mathrm{Ra}^{\mathrm{O}}=0,27 \mu \mathrm{m} ; \mathrm{Ra}^{\mathrm{nc}}=0,39 \mu \mathrm{m}$. c) $R_{a}{ }^{0}=0,09 \mu \mathrm{m} ; \operatorname{Ra}^{\mathrm{nc}}=0,23 \mu \mathrm{m}$. d) $\operatorname{Ra}^{0}=0,02 \mu \mathrm{m}$; $\operatorname{Ra}^{\mathrm{nc}}=0,13 \mu \mathrm{m}$.

Figure 2. Photomicrographs of different surface textures in nitrocarburized specimens. Before $\left(R a^{0}\right)$ and after $\left(R a^{n c}\right)$ the nitrocarburizing treatment. a) $R a^{0}=1.54 \mu \mathrm{m} ; R a^{n c}=1.72$ $\mu m$. b) $R a^{0}=0.27 \mu m ; R a^{n c}=0.39 \mu m$. c) $R a^{0}=0.09 \mu m$; $R a^{n c}=0.23 \mu \mathrm{m}$. d) $R a^{0}=0.02 \mu \mathrm{m} ; R a^{n c}=0.13 \mu \mathrm{m}$.

repitieron dos de las condiciones de ensayo, confirmándose la bondad de los resultados obtenidos.

Los resultados obtenidos guardan analogía con los datos existentes para procesos de nitrocarburación en baños de sales ${ }^{[4}$ 5], en los que la capa de combinación, con rugosidades del acero base de Ra $0,4 \mu \mathrm{m}$, presenta una zona externa porosa con una resistencia al desgaste menor que el resto de la capa. Los espesores de la capa porosa podrían esti-

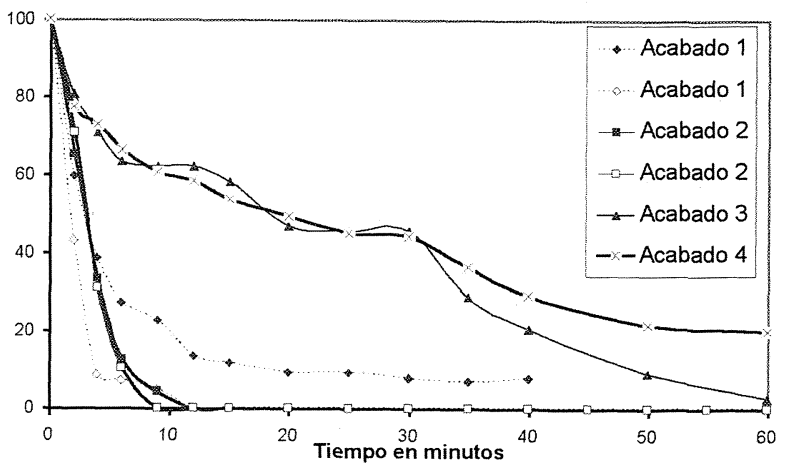

Figura 3. Espesor de capa desgastada en función del tiempo de ensayo. Los resultados se expresan en relación al espesor inicial de la capa de combinación o capa blanca.

Figure 3. Layer thickness lost versus time test. The results are expressed related to the initial white layer thickness.

marse, de acuerdo con los ensayos, con los valores establecidos.

En cualquier caso, se observa que los acabados más bastos conducen, en general, a mejores comportamientos frente al desgaste. La mayor resistencia al desgaste para los mayores valores de la rugosidad pueden justificarse en base a los mecanismos de atrapamiento de lubricante con tendencia a regímenes hidrodinámicos en lugar de lubricación límite $^{[7]}$.

\section{CONCLUSIONES}

El acabado superficial del acero, para los valores de rugosidad considerados, no parece condicionar la formación de capas nitrocarburadas si se asegura una atmósfera de gases suficiente en la proximidad de la superficie del material. La rugosidad de las muestras siempre aumenta con el tratamiento de nitrocarburación. El incremento en los parámetros de rugosidad se mantiene prácticamente constante en la gama de acabados superficiales experimentados, lo que significa en términos relativos que la variación en los acabados más finos es mucho más importante.

El comportamiento frente al desgaste abrasivo de la capa de combinación o capa blanca se caracteriza por una primera etapa en la que la pérdida de capa se produce a una velocidad más elevada que en el resto del ensayo. Además, la velocidad de desgaste en esta primera etapa es uniforme. Para los acabados más finos el espesor de capa con el comportamiento descrito es mayor, hasta un $90 \%$.

A partir de un determinado espesor de capa de combinación, que depende del acabado superficial 
Influencia del acabado superficial sobre el comportamiento tribológico de capas nitrocarburadas en acero X40CrMoV5 1

V. Miguel, A. Calatayud, J. Coello, A. Martínez y A. Caminero

del acero, la efectividad del resto de capa frente al desgaste es baja. En cualquier caso, los acabados superficiales más bastos conducen a una mejor resistencia al desgaste, con espesores menores de capa poco efectiva.

Los resultados obtenidos revelan que un mejor acabado superficial en la fabricación de matrices de extrusión no mejora, necesariamente, el comportamiento frente al posible desgaste abrasivo.

\section{REFERENCIAS}

[1] A. Calatayud, A. Martínez, J. Coello y V. Miguel, $\mathrm{V}$ Cong. Iberoam. Ingeniería Mecánica CIBEM6, Mérida,
Venezuela 2001. Dr. Simón Figueroa y Dr. Rigoberto Reinoza (Eds) pp. 531-536.

[2] M. MARTíneZ, Deformación metálica, 219 (1995) 23-35.

[3] A. Calatayud, J. Coello A. Martínez y V. Miguel, Vi Cong. Iberoam. Ingeniería Mecánica CIBEM6, Vol 1, A. M. Días (Ed) pp. 529-534.

[4] Y.H. QuiAng, S.R. Ge Y Q.J. XuE, J. Mater. Proces. Technol, 101 (2000) 180-185.

[5] Y.H. QIANG, S.R. GE Y Q.J. XUE, Wear 218 (1998) 232-236.

[6] A. MASEn, Ph. D. Thesis. University of Twente, Enschede, 2004.

[7] J.A. SCHEY, American Society for Metals. Metals Park, Ohio 44073, USA, 1983, p. 86. 